\title{
Dynamics of Microbial Growth and Coexistence on Variably Saturated Rough Surfaces
}

\section{Journal Article}

Author(s):

Long, T.; Or, Dani

Publication date:

2009

Permanent link:

https://doi.org/10.3929/ethz-b-000021138

Rights / license:

In Copyright - Non-Commercial Use Permitted

Originally published in:

Microbial Ecology 58(2), https://doi.org/10.1007/s00248-009-9510-3 


\title{
Dynamics of Microbial Growth and Coexistence on Variably Saturated Rough Surfaces
}

\author{
Tao Long $\cdot$ Dani Or
}

Received: 11 August 2008 / Accepted: 11 March 2009 /Published online: 8 April 2009

(C) Springer Science + Business Media, LLC 2009

\begin{abstract}
The high degree of microbial diversity found in soils is attributed to the highly heterogeneous pore space and the dynamic aqueous microenvironments. Previous studies have shown that spatial and temporal variations in aqueous diffusion pathways play an important role in shaping microbial habitats and biological activity in unsaturated porous media. A new modeling framework was developed for the quantitative description of diffusiondominated microbial interactions focusing on competitive growth of two microbial species inhabiting partially saturated rough surfaces. Surface heterogeneity was represented by patches with different porosities and water retention properties, yielding heterogeneous distribution of water contents that varies with changes in relative humidity or soil matric potential. Nutrient diffusion and microbial growth on the variably hydrated and heterogeneous surface was modeled using a hybrid method that combines a reaction diffusion method for nutrient field with individual based model for microbial growth and expansion. The model elucidated the effects of hydration dynamics and heterogeneity on nutrient fluxes and mobility affecting microbial population growth, expansion, and coexistence at
\end{abstract}

T. Long

Department of Civil and Environmental Engineering,

Tufts University,

Medford, MA 02155, USA

e-mail: tao.long@tufts.edu

D. Or $(\bowtie)$

Department of Environmental Sciences (D-UWIS),

Institute of Terrestrial Ecosystems (ITES),

Soil and Terrestrial Environmental Physics (STEP), ETH Zurich,

Zurich, Switzerland

e-mail: dani.or@env.ethz.ch the microscale. In contrast with single species dominance under wet conditions, results demonstrated prolonged coexistence of two competing species under drier conditions where nutrient diffusion and microbial movement were both limited. The uneven distribution of resources and diffusion pathways in heterogeneous surfaces highlighted the importance of position in the landscape for survival that may compensate for competitive disadvantages conferred by physiological traits. Increased motility was beneficial for expansion and survival. Temporal variations in hydration conditions resulted in fluctuations in microbial growth rate and population size. Population growth dynamics of the dominant species under wet-dry cycles were similar to growth under average value of diffusion coefficients for dry and wet conditions, respectively, suggesting that the timeaveraged diffusion coefficient could serve as a useful indicator for estimation of microbial activities in a highly dynamic system such as that found in soils.

\section{Introduction}

Soil is a complex and heterogeneous environment comprising of a mixture of solid, gaseous, and aqueous phases that are in a constant state of change and support a wide array of biological activities. Evidence suggests that the top soil layer represents the richest habitat for microbial life on Earth [9]. Notwithstanding the great bioactivity and the remarkable diversity hosted in this important compartment of the biosphere [32], our understanding of the origins and maintenance of soil microbial diversity remains limited. Microbial communities inhabiting natural soils are influenced by resource distributions, pore space structure and hydration conditions affecting mass and energy transport, interactions among competing species, and numerous other 
environmental factors [24]. Competing microbial species in homogeneous environments under steady inputs do not typically coexist [14]. Coexistence and microbial diversity are sustained by spatial heterogeneity, temporal variations in resource availability, and complex biogeochemical soil processes [13, 19, 33].

Soil aqueous phase exhibits spatial and temporal variations that exert significant influence on formation and resupply of microbial aquatic niches. Experiments [34] clearly showed that coexistence of two microbial species was suppressed under high soil matric potential (wet conditions). Evidence suggests that microbial diversity in the saturated zone is relatively lower than that found in unsaturated near-surface soils $[37,38]$. Limitations to microbial activity and movement under low water contents, combined with limited nutrient diffusion, may suppress the emergence of a dominant species [24, 34].

Direct observations of concurrent changes in soil aqueous phase and microbial activity are rare and difficult to obtain [11]. We thus consider studies on rough surfaces as useful theoretical and experimental research platforms for study of microbial growth and coexistence. Such two-dimensional (2-D) porous media offer continuous observability while maintaining complex configurations of aqueous phase diffusion pathways and overall water retention characteristics similar to that found in unsaturated soil [20,31]. Nevertheless, even for the simplest scenario of 2-D rough surfaces with no convective fluid flow, the interactions between surface heterogeneity, aqueous phase configuration, nutrient transport, and microbial motion and activity remain complex. Our objective was to develop a simplified modeling framework to elucidate temporal aspects of aqueous diffusion-mediated interactions affecting microbial activity and coexistence.

The proposed modeling approach link microhydrology with microbial activity and coexistence at the colony scale using hydraulic parameters upscalable to soil sample scale. Linking abiotic factors (hydration, heterogeneity, diffusion fluxes) with growth and coexistence of competing microbial species offer insights into the origins and mechanisms that maintain the unparalleled prokaryotic diversity found in soils. The results are expected to assist with the design of future experiments with prescribed and natural rough surfaces as observable porous media analogs [11]. The proposed model was designed to retain salient physical processes related to dynamic changes in water retention similar to those occurring in the shallow vadose zone (unsaturated zone) and quantify impacts on microbial growth and competition. Simplifications of environmental and physiological parameters inevitably cause inaccuracy; hence, this study is expected to throw some insight onto trends in microbial-environmental interactions and subject to future examination.

\section{Methods}

Rough Surface Representation

The amount of water stored within a soil or rock surface roughness and its spatial configuration vary predictably with changes in matric potential (or relative humidity of ambient air). A complete description of detailed roughness geometries is prohibitively complex; hence, a certain degree of simplification and abstraction is necessary. Various approaches have been used to represent rough surfaces ranging from surface fractal geometry [7, 27], simple root mean square of height differences (c.f., Tokunaga et al. [31]), and geometrical abstraction using simple geometrical elements [25]. In a previous study [20], we addressed the distribution of transport pathways on a rough surface by assembling simple geometrical elements into a network. The primary limitations of such detailed surface roughness representation lies in the heavy computational burden required for resolving the temporal changes in the aqueous and nutrient fields, the extensive parameterization requirements, and the limited prospects for upscaling such a model beyond a few square millimeters. Hence, while such representation may guide the construction of artificial rough surfaces for future experiments, understanding of the processes at larger scales (larger than square centimeters) requires a simpler representation of rough surfaces.

We proposed an alternative representation of natural rough surfaces based on using discrete patches with prescribed porosity and water retention behavior that maintained the spatial heterogeneous structure and water retention properties of the surface. Within each unit area, the patch had uniform roughness which was represented by prescribed porosity and water retention parameters. The distribution of aqueous phase on the entire rough surface (simulation domain) under a given matric potential value was determined by the spatial distribution and the water retention characteristics of the individual patches. All other processes, such as nutrient diffusion and microbial growth, were modeled based on the resulting aqueous phase distribution. The patchedsurface approach was amenable for scaling up towards quantifying microbial growth and activity in real porous medium at sample scale, if a valid representative patch size can be used to discrete the surface. The patch size of $0.2 \times$ $0.2 \mathrm{~mm}$ was used in this investigation.

\section{Water Retention on a Patched Rough Surface}

Hydration status and roughness for the patched-surface model were linked via water retention parameters for individual roughness patches. A considerable body of literature suggested that water film thickness as a function of matric potential could be represented as a power law 
$[6,31]$, and such representation was compatible with wellestablished parametric models for water retention in threedimensional porous media such as the Brooks and Corey [5] model as illustrated by Liu [18] for fractured rock surfaces. To represent the water retention properties of a patch, we used a widely adopted parametric model proposed by van Genuchten [36] that related saturation and matric potential according to:

$\Theta=\frac{\theta-\theta_{r}}{\theta_{s}-\theta_{r}}=\left[\frac{1}{1+\left(\alpha\left|\psi_{\mathrm{m}}\right|\right)^{n}}\right]^{m}$

where $\theta$ is the volumetric water content of the porous medium (rough surface), $\theta_{\mathrm{s}}$ is the volumetric water content at saturation (also equals rough surface porosity $\Phi$ ), $\theta_{\mathrm{r}}$ is the residual water content (due to adsorbed film thickness under very dry conditions - see Tuller and Or [35]), $\psi_{\mathrm{m}}$ is the matric potential, $\alpha$ is related to the air entry value in a porous medium or to the matric potential value at which largest roughness elements begin to empty, and $m$ and $n$ are empirical parameters related to roughness properties and interrelated by $m=1-1 / n$. The rough surface porosity is considered as the volume of water stored within the surface roughness under zero matric potential (deduced from geometrical interpretation or by direct measurements such as those reported in [31])

The simulations reported herein were based on the van Genuchten parameters for an equivalent silt loam soil reported by Or and Hanks [23] and listed in Table 1. A rough surface domain with spatial dimensions of $10 \times$ $10 \mathrm{~mm}$ was discretized to form $50 \times 50$ square patches (grid cell size $\Delta x=\Delta y=0.2 \mathrm{~mm}$ ). Each patch was randomly assigned with van Genuchten retention parameters drawn from log-normal distribution with mean values and prescribed variance as given in Table 1 . In the absence of experimental data for soil properties or surface roughness at such small scales, the log-normal distribution associated variances were chosen arbitrarily. Similarly, no specific spatial correlation was prescribed in the generation of the rough surfaces. Nevertheless, introducing variances in patch properties was a critical step to forming heterogeneous surfaces at scales important for microbial activity and interactions. We thus focused in this study on microbial

Table 1 Parameters used to create rough surfaces

\begin{tabular}{lcc}
\hline Parameters & $\begin{array}{l}\text { Values for HM soil } \\
\text { (mean values for HT soil) }\end{array}$ & $\sigma^{2}$ for HT soil \\
\hline$\theta_{\mathrm{s}}\left(\mathrm{m}^{3} \mathrm{~m}^{-3}\right)$ & 0.513 & 0.5 \\
$\theta_{\mathrm{r}}\left(\mathrm{m}^{3} \mathrm{~m}^{-3}\right)$ & 0.05 & 0.05 \\
$\alpha\left(\mathrm{m}^{-1}\right)$ & 0.417 & 0.01 \\
$n$ & 1.75 & 0.1 \\
\hline
\end{tabular}

growth, expansion, and coexistence on a heterogeneous (HT) and an equivalent homogeneous (HM) surface (no variance was assigned to patch properties) to contrast responses due to spatial heterogeneity.

Surface porosity or saturated water content $\left(\theta_{\mathrm{s}}\right)$ and residual water content were constrained for physical consistency (assuming that the maximum porosity of a patch was less than $90 \%$ for the roughest surface and $\theta_{\mathrm{r}}<\theta_{\mathrm{s}}$ for the same patch). The local water content $\theta$ for a given matric potential was calculated by the van Genuchten model (Eq. 1), and used to construct the equilibrium configuration of the aqueous phase within the entire simulated rough surface.

\section{Nutrient Diffusion within a Patched Rough Surface}

Nutrient transport within the aqueous phase was numerically simulated using the finite difference method (FDM). The nodes of the FDM grid were set at the centers of each roughness patch. The continuous form of the governing equation for the system is:

$\frac{\partial N}{\partial t}=\nabla \times D \nabla N-R(x, y, t)$

where $N$ is the concentration of nutrient [in nanograms per cubic millimeter], $D$ is the local aqueous diffusion coefficient [in square millimeters per hour], and $R$ [in nanograms per cubic millimeter second] is the local consumption rate of nutrient. The effective diffusion coefficient in unsaturated rough surface $\left(D_{\mathrm{S}}\right)$ is a function of surface porosity and water content and was estimated by the so-called Millington and Quirk (MQ) model [21], which requires the nutrient diffusion coefficient in water $D_{0}$ as the only additional parameter:

$D_{\mathrm{s}}=\frac{D_{0} \theta^{10 / 3}}{\theta_{\mathrm{s}}^{2}}$.

Based on the spatial discretization scheme, $D$ values were determined at boundaries of patches by substituting mean $\theta$ values of each bordering patch pairs into Eq. 3. The microbial nutrient consumption rate $R(x, y, t)$ is defined as the sum of consumption rates in each grid cell $(\Delta x \times \Delta y)$ during a time step $\Delta t$ (or the amount of available nutrient when nutrient is insufficient to satisfy the expected consumption). Evolution of the system is solved with the alternate direction implicit method [26].

Simulation of Microbial Activity and Motility on Patched Rough Surfaces

The quantitative and spatially explicit BacSim model [17] was used to simulate microbial growth in this study. 
BacSim is an individual based model that describes microbial activity at the individual cell level, including substrate uptake, metabolism, maintenance, reproduction, and decay. Michaelis-Menten type growth has been assigned to individual cells as shown in Eq. 4, which guarantees Monod type of growth of the total population:

$-V \frac{\mathrm{d} s}{\mathrm{~d} t}=v=\frac{x V_{\max } s}{K_{\mathrm{m}}+s}$

where $V$ is the aqueous volume of the lattice grid, $s$ is the substrate concentration, $v$ is the rate of substrate uptake (mass/time), $x$ is the cell dry mass of the individual cell, and $V_{\max }$ is the maximum specific substrate uptake rate $\left(V_{\max }=\mu_{\max } / Y_{\max }\right.$, glucose $/($ dry mass $\times$ time $\left.)\right)$. It is important to notice that this equation applies to individual cells. Parameters for the microbial component of the model are given in Table 2. Details of the BacSim model can be found in Kreft et al. [17]. Only simple resource competition [14] was considered between two virtual species to enhance understanding of microbial coexistence.

Tilman [30] addressed the importance of the threshold resource level $\left(R^{*}\right)$ for the evaluation of competition capacity of species for limited resources. $R^{*}$ is the nutrient concentration at $\mu=0$, which can be calculated by:

$R^{*}=\frac{K_{\mathrm{S}} r_{\mathrm{m}} Y_{\max }}{\mu_{\max }-r_{\mathrm{m}} Y_{\max }}$.

If resource concentration remains below the threshold resource level $R_{i}^{*}$ for a specific species $i$, population density will decrease and extinction is inevitable. In the scenario of microbial competition for a single resource, when resource supply is limited, a species with positive growth rate at the lowest $R^{*}$ will exclude all other species [30]. In the set of parameters chosen in Table 2, the only physiological difference between SP1 and SP2 is given by lower $K_{\mathrm{s}}$ for SP1 conferring competitive advantage over SP2 ultimately excluding SP2 in homogeneous environments under limited nutrient supply. Moreover, as the maximum specific growth rates and starvation time for both species were identical, SP2 would not survive competition with SP1 at steady state in a well-mixed environment. In other words, SP1 and SP2 did not form a "gleaner" (lower $R^{*}$ and also lower $\mu_{\max }$ ) and "exploiter" (vice versa) couple [14] and could not take turns to thrive under alternating poor and rich nutrient supplies.

Microbial motility was another critical factor at the microscale considered in this study with no convective flow [24]. Microbes move on surfaces by a variety of means such as swimming, swarming, gliding, twitching, sliding, and darting [15]. The specific mechanism and rate of motion of a species are determined by functional cell appendages (flagella, pili, etc.) and regulated by extracellular signals transmitted within the population. Complicated biological and physical processes at pore scale are involved in microbial motility near surfaces, such as the confinement of pore space for swimming bacteria [3, 22]. Abundance of water (or effective water film thickness) is critical for bacterial swimming and also promotes cell motility by swarming, twitching, and gliding $[2,15]$. Clearly, the full impact of water content and configuration on various modes of microbial motility cannot be generalized at this state of knowledge. However, we applied heuristic arguments for our numerical modeling that would capture the primary effects of rough surface water content on microbial translocation, without explicitly considering the full complexity of motility in confined aqueous phase (e.g., [8]). When average water film thickness on a patch was greater than a prescribed threshold $(0.1 \mathrm{~mm}$ in the simulations), we assigned $100 \%$ probability for the cell movement to occur within the patch, otherwise translocation probability decreased linearly with average water film thickness. The role of such critical mobility threshold on spreading and maintenance of coexistence was a subject of a study by Reichenbach et al. [28]. Microbial movement to a neighboring patch was dependent on average water content of both patches. The translocation distance of a microbe was simply expressed as a random fraction of the product of maximum motility (as shown in Table 2) and time step length; and the direction of movements in each time step was randomly generated to represent observed changes in directions for motile species [10].
Table 2 Biological parameters used in the hybrid model

${ }^{\mathrm{a}} 1 \mathrm{fg}=10^{-15} \mathrm{~g}$

\begin{tabular}{lllc}
\hline Parameters & Units & SP1 & SP2 \\
\hline$\mu_{\text {max }}:$ specific maximum growth rate & $\mathrm{h}^{-1}$ & 1.23 & 1.23 \\
$K_{\mathrm{s}}$ : half-saturation constant & $\mathrm{fg} \mathrm{fl}^{-1 \mathrm{a}}$ & $1.17 \times 10^{-6}$ & $2.34 \times 10^{-6}$ \\
$Y_{\text {max }}:$ apparent yield at $\mu_{\max }$, corrected & fg dry mass/fg glucose & 0.44 & 0.44 \\
$\quad$ for maintenance & fg glucose/(fg dry mass h) & 0.18 & 0.18 \\
$r_{\mathrm{m}}:$ apparent maintenance rate at $\mu=0$ & $\mathrm{fl}$ & 0.4 & 0.4 \\
$V_{u}:$ median cell volume at $\mu=0$ & $\mathrm{fg} \mathrm{fl}^{-1}$ & 290 & 290 \\
$\rho:$ cell density (dry mass) & $\mathrm{mm} \mathrm{h}^{-1}$ & 0.2 & 0.1 \\
$\mathrm{r}:$ motility & & & \\
\hline
\end{tabular}




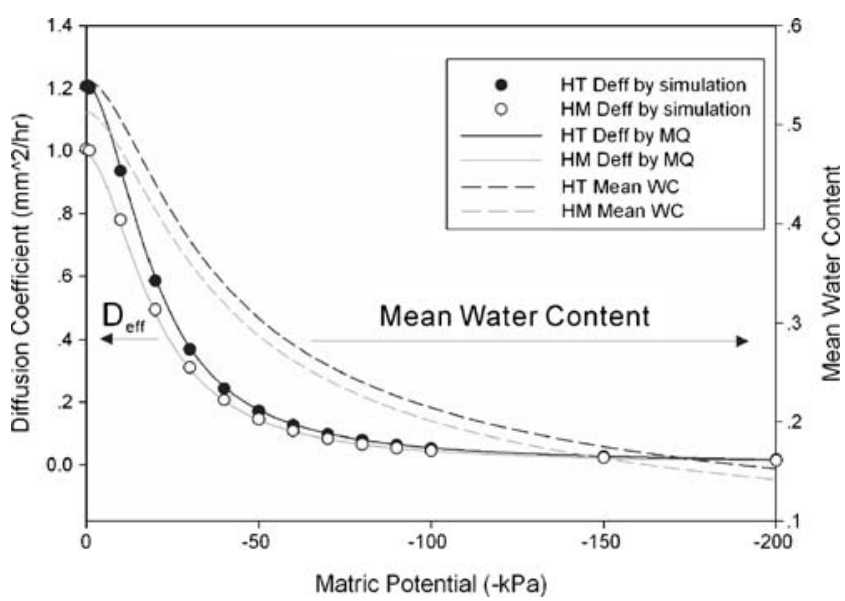

Figure 1 Mean volumetric water contents (dashed curves) and effective diffusion coefficients estimated by the MQ model (solid curves) for partially saturated rough surfaces as a function of matric potential. Circles represent estimated effective diffusion values from numerical tests on heterogeneous (HT) and homogeneous (HM) rough surfaces

Due to the limited size of the simulated domain and continuous nutrient supply at the boundaries, we restricted the total simulation time to $150 \mathrm{~h}$ to avoid microbial expansion to the boundaries. For each simulation scenario employing a specific rough surface and a prescribed matric potential variation scheme, we performed five parallel growth simulations. The growth curves and coexistence times in each simulation varied due to the incorporation of random parameters, and the averaged population dynamics of the five replicates were recorded and used for comparison with other scenarios.

\section{Results}

Effects of Variation of Hydration Status on Solute Diffusion

Effective nutrient diffusion coefficient for unsaturated porous media is typically determined by steady-state experiments where a constant concentration gradient is imposed through a porous column and the diffusant fluxes are measured at the boundaries [1]. Based on these principles, we conducted numerical experiments to characterize the effective diffusion coefficients for homogeneous (HM) and heterogeneous (HT) unsaturated rough surfaces using radial diffusion from the boundaries to a sink in the center. A constant concentration $C_{0}$ of the diffusant was maintained at the boundaries of the square domain, whereas zero concentration was maintain at the center (acting as a continuous sink). Maintenance of constant concentrations required the addition or removal of nutrients at each time step. The amounts of which were denoted as $\Delta M_{1}(t)$ and $\Delta M_{2}(t)$. When the difference between $\Delta M_{1}(t)$ and $\Delta M_{2}(t)$ became negligibly small, the diffusion process was assumed to have reached steady state. The solute diffusion coefficient in pure aqueous phase used in the simulations was $D_{0}=2.4 \mathrm{~mm}^{2} / \mathrm{h}[17]$.

The steady-state concentration profile on the rough surface was compared to that developed in a cylindrical system [4]:

$\frac{\partial C}{\partial t}=D_{\mathrm{e}}\left(\frac{\partial^{2} C}{\partial r^{2}}+\frac{1}{r} \frac{\partial C}{\partial r}\right)$

where $C$ is the solute concentration, $r$ is the radial distance from the center, and $D_{\mathrm{e}}$ is the effective diffusion coefficient. Analytical concentration distribution curves were fitted to steady-state concentration profiles obtained from the numerical experiments. The numerical concentration profiles were used to estimate the effective aqueous diffusion coefficient for the partially saturated rough surfaces under different hydration states.

In practice, $D_{\mathrm{e}}$ values for partially saturated porous media are estimated by means of a parametric model such as that described by Eq. 3 [21] based on mean water content of the rough surface $(\theta)$. Effective diffusion coefficient values obtained by both methods under different matric potentials (and saturations) were plotted in Fig. 1, including the water retention properties expressed as mean water
Figure 2 The spatial distribution of the aqueous phase on the rough surface under -0.01 and $-100 \mathrm{kPa}$ matric potential. The scales of the color map represent the volumetric water contents. The red square represents the simulation domain for the results depicted in Figs. 3, 5 (columns a and b), 6 and 8 . The dimensions of the entire surface are $10 \times 10 \mathrm{~mm}$

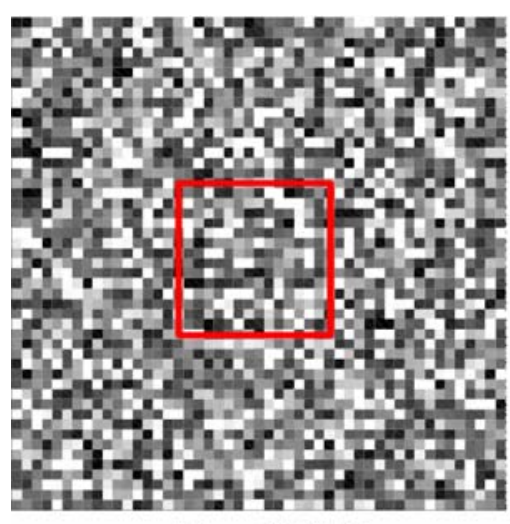

$\Delta \mathrm{P}=-0.01 \mathrm{kPa}$
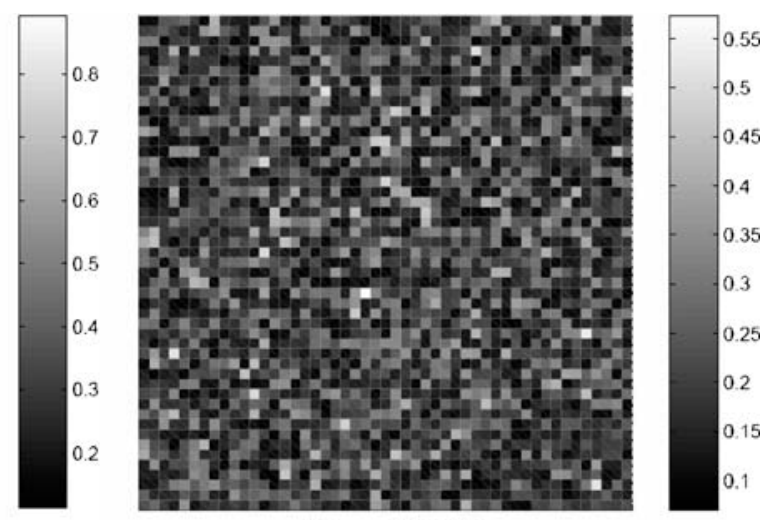

$\Delta \mathrm{P}=-100 \mathrm{kPa}$ 
content retained within the rough surface. The results showed a decrease in $D_{\mathrm{e}}$ with decreased matric potential (more negative). Furthermore, $D_{\mathrm{e}}$ values obtained from numerical tests were in good agreement with those calculated from the empirical MQ model, confirming the consistency of the numerical scheme.

A homogeneous surface of the same size was also generated by assigning the mean parameters in Table 1 to all the patches (no variance). Theoretically, homogeneous surfaces ensured that the mean effective diffusion coefficient of the surface was spatially uniform. We observed a close agreement between numerically obtained $D_{\mathrm{e}}$ values and MQ model estimates [21] for the homogeneous surface as depicted in Fig. 1, ensuring consistency of the numerical diffusion scheme and its capability to scale up diffusion processes from a single patch to the entire surface. The effective diffusion coefficients for heterogeneous surface were not significantly different from those of the homogeneous surface because $D_{\text {eff }}$ represented an aerial average over the whole surface, the retention properties of which were governed by the same mean values. In other words, mean water contents were similar for the homogeneous and heterogeneous surfaces (dashed lines in Fig. 1) for a given matric potential irrespective of the random spatial heterogeneity.

\section{Microbial Growth and Competition Under Constant Matric Potential Values}

Numerical simulations of microbial growth and competition were performed on the HM and HT patched rough surfaces (with patch sizes of $200 \times 200 \mu \mathrm{m}$ ). Different values of ambient matric potential were imposed, resulting in different contents and distributions of the aqueous phase, as shown in Fig. 2 for $-0.01 \mathrm{kPa}$ (wet) and $-100.0 \mathrm{kPa}$ (dry) conditions. For each aqueous phase distribution, the hybrid model for nutrient diffusion and microbial growth
Figure 3 Results of the HM, HT, and HT-S simulations, showing microbial distribution after $150 \mathrm{~h}$ of elapsed simulation time under four different matric potential values. Gray dots represent SP1 cells, and the red dots represent SP2 cells. The symbols "+" and " $\times$ " indicate initial inoculation sites

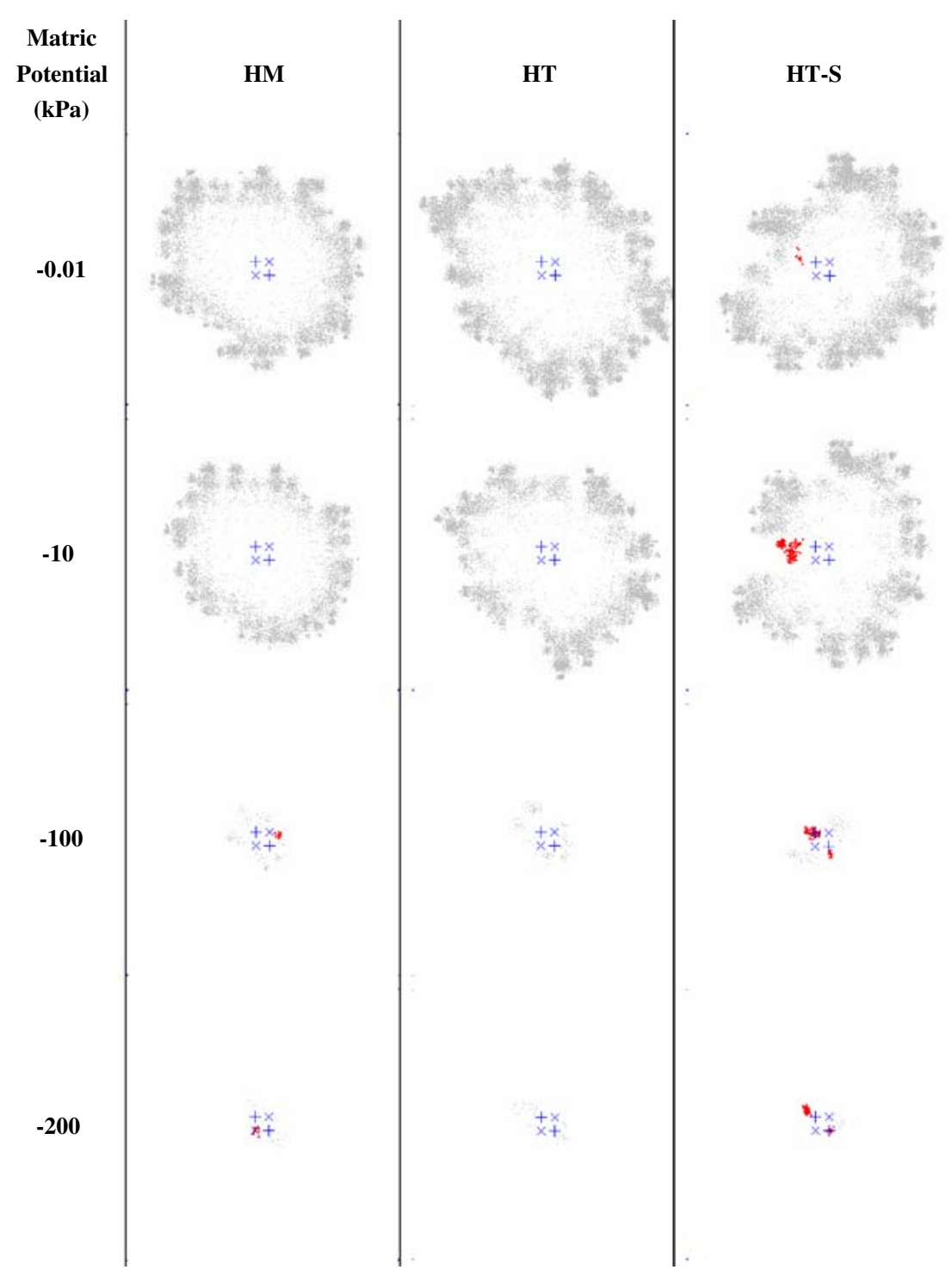


were applied to simultaneously simulate microbial activity and nutrient dynamics. In the first two simulation series, the HM and HT surfaces were used respectively with the same inoculation pattern. Each simulation was initiated by inoculating four sites in the central area of the rough surface, located at the corners of a $200 \times 200 \mu \mathrm{m}$ square as shown in Fig. 3. SP1 (the stronger competitor) was inoculated at the upper left (UL) and lower right (LR) points, while the weaker SP2 took the upper right (UR) and lower left (LL) points. We performed a special simulation (denoted as simulation HT-S) to examine the effects of switching of initial inoculation points on competition. The biological parameters of each species were given in Table 2 . The initial nutrient concentration in the aqueous phase was uniform at $0.2 \mathrm{mg} / \mathrm{L}$ everywhere. Subsequently, nutrient concentration at the domain boundaries was maintained constant at $0.2 \mathrm{mg} / \mathrm{L}$. Microbial growth and coexistence simulations were carried out under -0.01 (near saturation), $-10,-100$, and $-200 \mathrm{kPa}$.

Microbial growth with competition was simulated in five replicates of $150 \mathrm{~h}$ under each matric potential value. The randomness in microbial motility lead to different growth and nutrient consumption patterns from the same initial conditions. The final locations of active cells for each scenario were shown in Fig. 3 (showing a replicate with the longest coexistence time). The averaged population dynamics of the five replicates were shown in Fig. 4. In all simulations, at least one species survives to the end, indicating that nutrient supply from the boundaries was sufficient to maintain adequate microbial activity. For wetter conditions, microbes migrated away from inoculation points (Fig. 3), demonstrating enhanced expansion rates and higher motility under wetter conditions as stipulated by the model.

Microbial growth under four different matric potential values shared certain features. Starting from inoculation points at the center of the rough surface, fronts of active cells spread outwards intercepting all nutrient fluxes and forming nutrient-depleted zones in the center. The active fronts of microbes were irregular in shape, reflecting combined effects of individual-based nature of microbial modeling scheme and spatial heterogeneity of the rough surface. Immediately after inoculation, we observed synchronized growth for cells belonging to the same species due to similar initial parameters and environmental conditions [17]. Subsequently, cell growth became less synchronized due to the differences in nutrient consumption and other local interactions experienced by individual cells.

The biological parameters for the study were selected to provide SP1 with competitive advantage by assigning a lower $K_{\mathrm{s}}$ value for SP1 which in turn promotes faster growth when substrate concentration was insufficient to support maximum growth rates for both species. Addition- ally, the higher motility of SP1 cells enhance their expansion rates and opportunities for moving to patches with more favorable nutrient supply. On the HM surface, the extinction of SP2 under -0.01 and $-10 \mathrm{kPa}$ could be attributed to the rapid spreading of SP1 fronts that cut off nutrient supply to the SP2 colonies. For dryer conditions
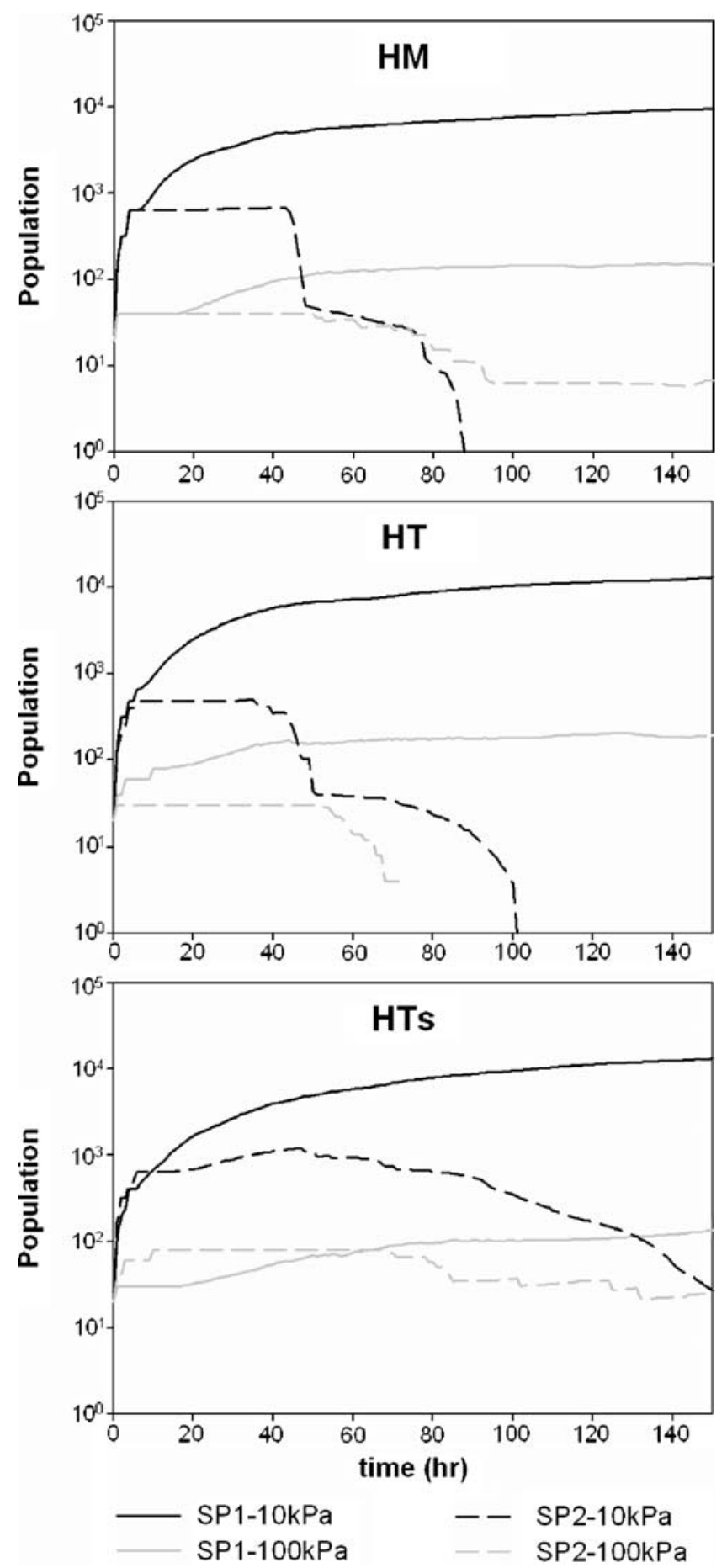

Figure 4 Averaged population growth curves of the HM, HT, and HT-S simulations for the -10 and $-100 \mathrm{kPA}$ scenarios demonstrated in Fig. 3 
$(-100$ and $-200 \mathrm{kPa})$ where both water content and total nutrient amount were limited, not only that the motility of SP1 was significantly reduced, but also its reproduction rate slowed down. The reduced spreading rates of the stronger competitor significantly prolonged periods of coexistence of the two species (Fig. 4a).

Even for the HM surfaces with initially uniform nutrient distribution, heterogeneities in nutrient distribution and fluxes arose due to nonuniform growth and expansion of microbial population as seen in Fig. 5. Microbial expansion, generally dominated by the stronger species SP1, was coupled with nutrient depletion starting at the inoculated center. Maximum microbial activity as well as maximum population size occurred at the boundaries of the expanding nutrient depletion zone. Under wet conditions with high motility, the stronger competitor SP1 spread out forming a nearly continuous front that quickly bypass SP2 clusters and cut off its nutrient supply by leaving it inside the nutrient-depleted zone. For dryer conditions, population density and motility of SP1 were greatly reduced, resulting in extended coexistence periods. In other words, higher water contents promote diffusive nutrient transfer and microbial motility, thereby enhancing bioactivity and expansion rate that, in turn, accelerate extinction of the weaker species.

\section{The Role of Heterogeneity}

Motility on the heterogeneous rough surface was also enhanced under wetter conditions; however, drier conditions did not always extended coexistence time, as evidenced by HT simulation results (Fig. 4b). The primary reason for this inconsistency is attributed to spatial heterogeneity of water contents that induce uneven resource abundance and form heterogeneous diffusion pathways. Spatially variable water content on the heterogeneous surface with uniform initial nutrient concentration implies proportionality of local nutrient mass with water content.
Figure 5 Evolution of microbial colony shape, population distribution of SP1, and nutrient concentration field with simulation time, as shown in columns $a, b$, and $c$. The results are for $-10 \mathrm{kPa}$ on the homogeneous (HM) rough surface. The red dots in column $a$ represent SP2 cells. Columns $a$ and $b$ represent the central domain of the rough surface as highlighted in Fig. 2. Column $c$ depicts nutrient distribution over the entire surface
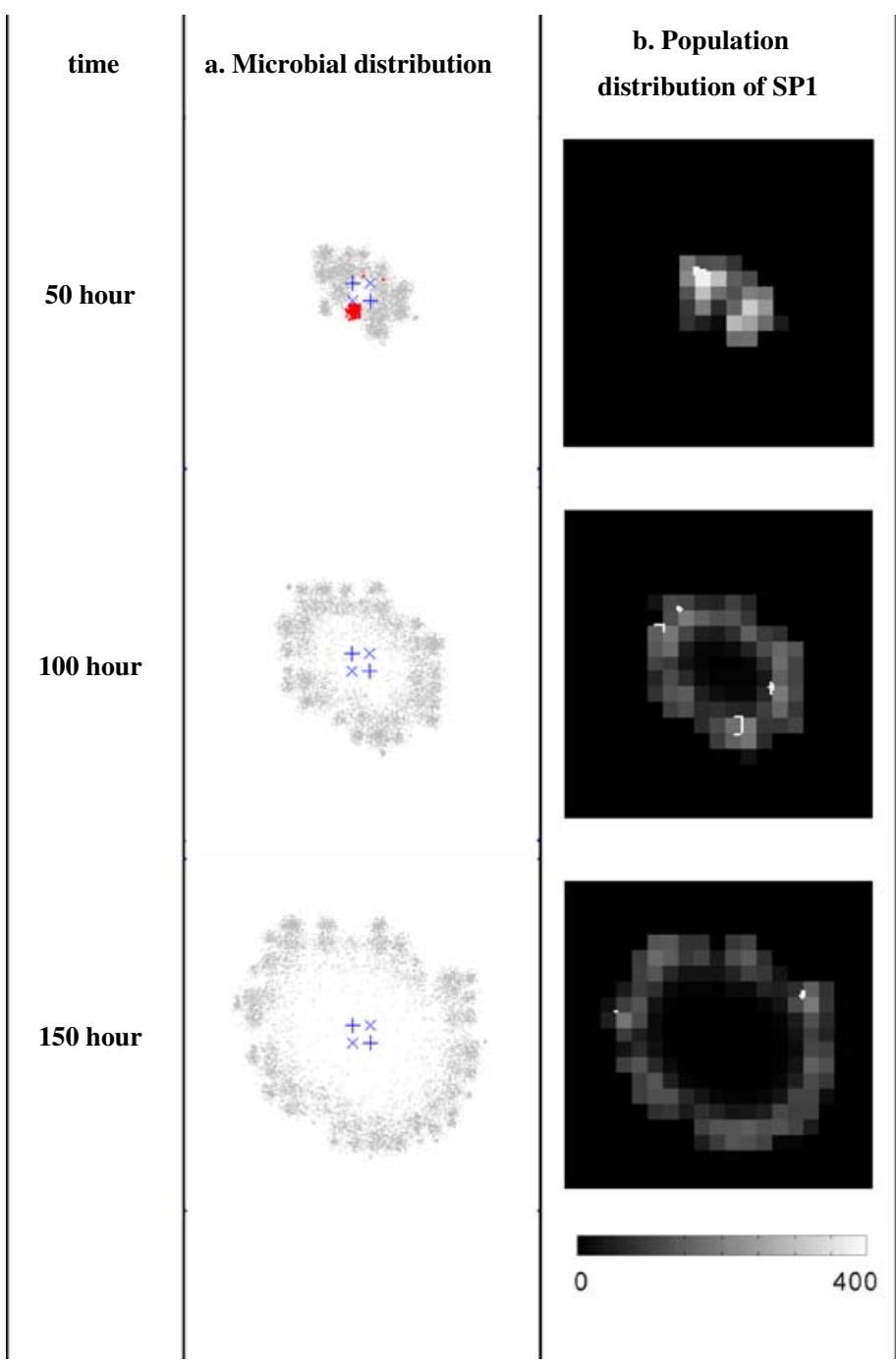

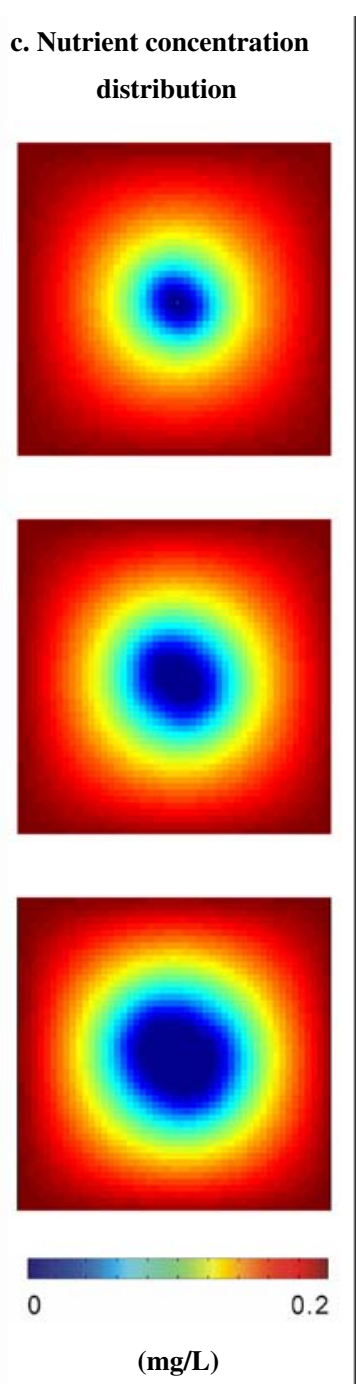

$(\mathrm{mg} / \mathrm{L})$ 
Patches with high water content promoted rapid initial growth of microbial population. For inoculated patches connected to the domain boundary (with constant nutrient concentration) through connected "wet" patches form preferential diffusion pathways that supply nutrients at higher mass transfer rates. However, such pathways were also invaded preferentially by microbes as motility was also enhanced with higher water content. Therefore, patches with more nutrient or connected to preferential pathways offer microbes rapid growth rates. This might explain how the short survival time of SP2 on HT could be significantly extended by switching inoculation locations with SP1 (simulation HT-S). However, initial nutrition could only boost population in a short term, and the pattern of preferential diffusion pathways evolved while microbes relocated themselves on the surface. Thus, the locations of patches providing growth advantages change dynamically with time and with microbial population distribution. Examples of colony evolution in the HT and HT-S simulations were provided in Fig. 6, highlighting the impact of spatial heterogeneity on microbial coexistence.

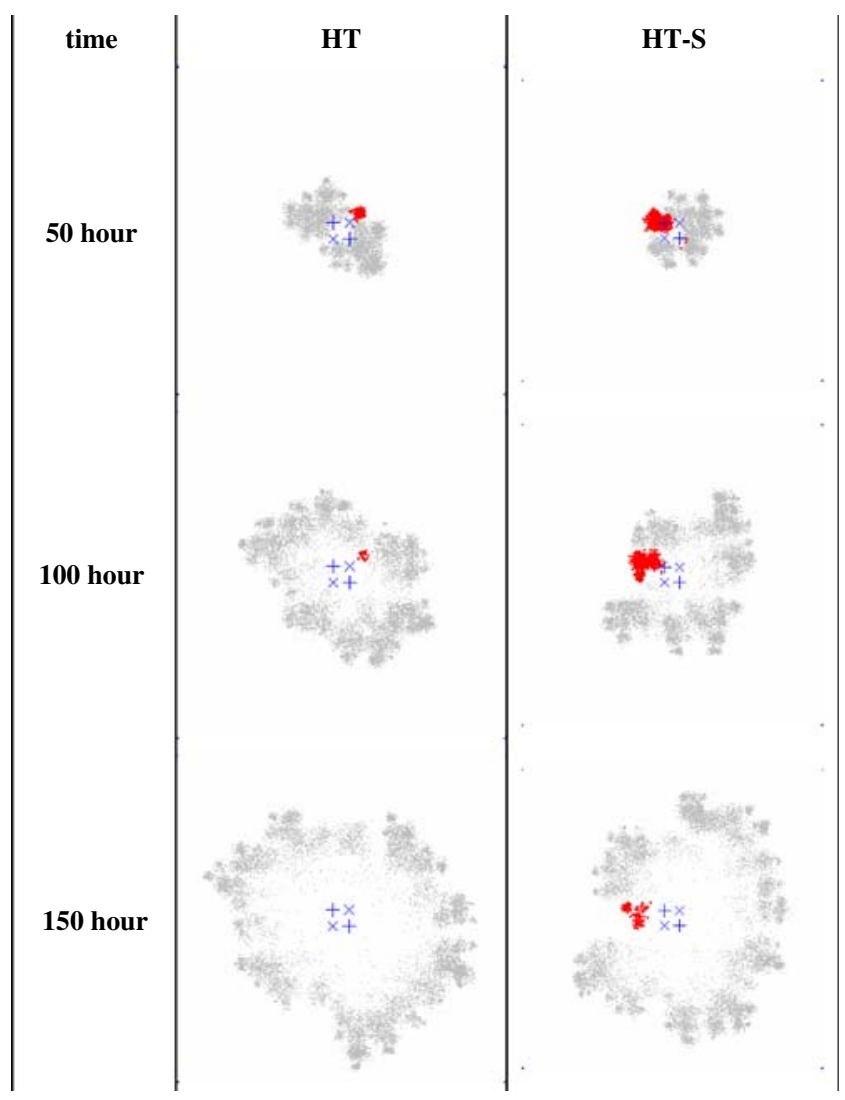

Figure 6 Evolution of microbial colony shape with simulation time in the HT and HT-S simulations. The gray dots represent SP1 cells, and the red dots represent SP2 cells. The size of each figure equals the central domain of the rough surface as highlighted in Fig. 3
To investigate the importance of spatial heterogeneity on overall protection of microbial diversity, 50 homogeneous and 50 different heterogeneous surfaces were randomly generated with parameters provided in Table 1. On each rough surface, 20 microbes of each species were inoculated randomly in the central $0.8 \times 0.8 \mathrm{~mm}$ region. The boundary conditions remained unchanged. Competition was simulated at $-10 \mathrm{kPa}$ for $150 \mathrm{~h}$ simulation time. Results show that, on the homogeneous surfaces, only seven out of 50 cases lead to SP2 survival at the end of $150 \mathrm{~h}$, while growth on heterogeneous surfaces yielded 15 cases out of 50 simulations. Figure 7 depicts SP2 survival times for all simulations showing a clear shift to longer coexistence time on HT surfaces. The results confirm the important role of spatial heterogeneity in sustaining microbial diversity in unsaturated soil.

\section{The Role of Motility}

As described previously, SP2 cannot coexist under the competitive pressure of SP1 in a well-mixed environment. On a rough surface, spatial distribution of nutrient concentrations among roughness patches made spatial location a potentially critical factor for local microbial growth and survival performance.

Maximum motility parameter for SP2 was originally chosen as $0.1 \mathrm{~mm} / \mathrm{h}$, while the stronger SP1 moved at $0.2 \mathrm{~mm} / \mathrm{h}$ (these are at low range of sliding-gliding motility values reported by Harshey [15]). To isolate and test the role of motility in microbial growth and survival, increased $(0.2 \mathrm{~mm} / \mathrm{h})$ and decreased $(0.05 \mathrm{~mm} / \mathrm{h})$ motilities of SP2 were used respectively for simulations on the HM surface, keeping all other parameters unchanged. Four different matric potential values were used, and five replicated simulations were carried out for each matric potential value. The distributions of microbes after $150 \mathrm{~h}$ of elapsed simulation time were shown in Fig. 8 (results from the replicate with longest SP2 survival time). The averaged population growth curves were given in Fig. 9.

Simulation results are compared with those obtained with original parameters on the HM surface (Figs. 4a and 5), indicating that reduced SP2 motility diminished its competitiveness for wetter conditions (matric potential value of $-10 \mathrm{kPa}$ ) resulting in a decrease in average survival (coexistence) time from about 70 to $45 \mathrm{~h}$. In contrast, increasing motility enhanced competitiveness of SP2 to levels similar to SP1, despite other physiological parameters indicating that SP2 was a weaker competitor. At low matric potentials $(-100 \mathrm{kPa})$, survival times were practically unaffected by variations in the motility parameter, suggesting that physical limitations imposed by fragmented aqueous phase distribution and reduced film thickness restricted microbial motion and expansion rates. These findings are 
Figure 7 Histograms of coexistence duration (in hours) obtained from random inoculation microbial growth simulations extending $150 \mathrm{~h}$ on homogenous surfaces (a) and heterogeneous surfaces (b). Seven out of 50 simulations performed on the HM surface resulted in survival of SP2 at the end of $150 \mathrm{~h}$, whereas in 15 out of 50 cases SP2 survived on the randomly generated HT surfaces. Detailed population growth curves of these simulations are shown in $\mathbf{c}$ for HM surfaces and d for HT surfaces with gray curves representing SP1 population growth and black curves representing SP2
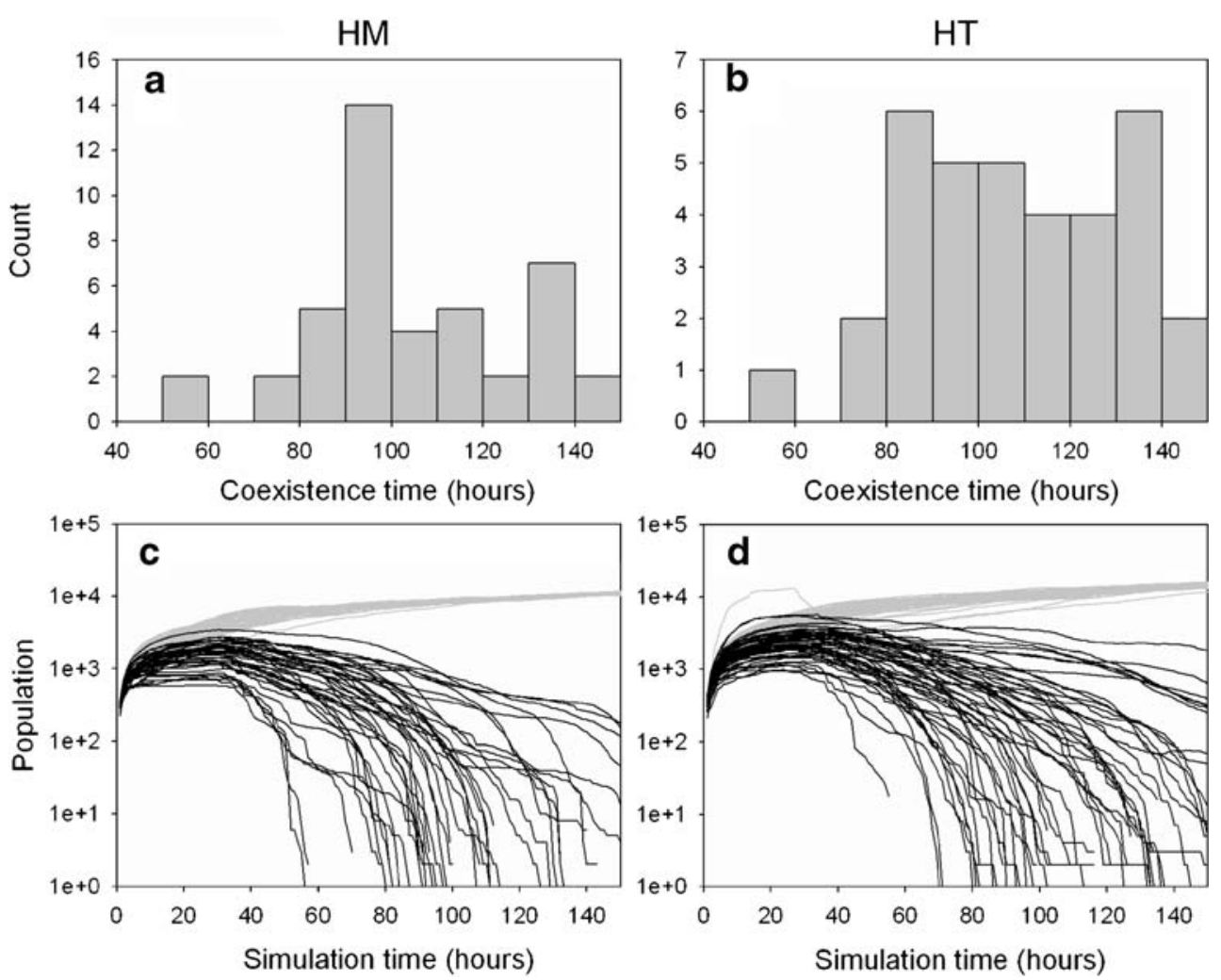

in agreement with recent studies concerning the role of motility in preserving or jeopardizing diversity within competing populations [28].

\section{Microbial Coexistence Under Temporal Variations in Matric Potential}

Simulation studies of microbial growth and coexistence on HM rough surface with similar initial and boundary conditions as in previous section, but with temporal variations in matric potential were performed. For simplicity, we induced alternating conditions between wet and dry periods $(-10$ to $-100 \mathrm{kPa}$ ) each lasting 10,20 , or $40 \mathrm{~h}$. Constant nutrient concentrations were maintained at the boundaries. The resulting population growth dynamics were plotted in Fig. 10.

The results in Fig. 10 show the significant impact of temporal variations in hydration conditions (matric potential) on growth rates of the dominant species (SP1). Fluctuations in SP1 population size were practically synchronized with matric potential oscillations, exhibiting steady growth during wet periods and stagnation (or even decay) during dry periods. These variations were attributed to reduced amounts of available nutrient and the limited microbial motility, both controlled by the matric potential. SP2 population size and growth was affected to a smaller extent by rapid fluctuations in matric potential (i.e., every 5 and $10 \mathrm{~h}$ ). The magnitude of population fluctuation for both
SP1 and SP2 increased with the increasing length of the dry and wet periods. Results indicate that the weaker competitor SP2 was unable to acquire sufficient nutrients during wet periods and was less adapted to surviving prolonged dry periods. The results in Fig. 10 suggest that longer durations of dry periods tend to reduce the survival time of SP2.

In search for an effective representation of temporal variations in matric potential on microbial growth and coexistence, we considered temporally averaged effective diffusion coefficient as a measure of diffusion capacity of the rough surfaces over extended time. For the wet-dry cycles, this coefficient was the mean of each effective diffusion coefficient weighed by the length of its duration:

$\bar{D}_{\mathrm{e}}=\frac{D_{\mathrm{e}, \mathrm{wet}} t_{\mathrm{wet}}+D_{\mathrm{e}, \mathrm{dry}} t_{\mathrm{dry}}}{t_{\mathrm{wet}}+t_{\mathrm{dry}}}$.

The resulting $\bar{D}_{\mathrm{e}}$ values for different wet-dry scenarios were calculated, and an effective matric potential representing similar diffusion conditions could be obtained from Eq. 3. The growth curves of SP1 under effective $\bar{D}_{\mathrm{e}}$ values (with its corresponding effective matric potential) were plotted with the growth curves for alternating matric potentials in Fig. 11. The growth curves with constant $\bar{D}_{\text {e }}$ values were capable of capturing the general growth tendency of various temporal variation schemes, including wet and dry periods of equal and nonequal lengths 


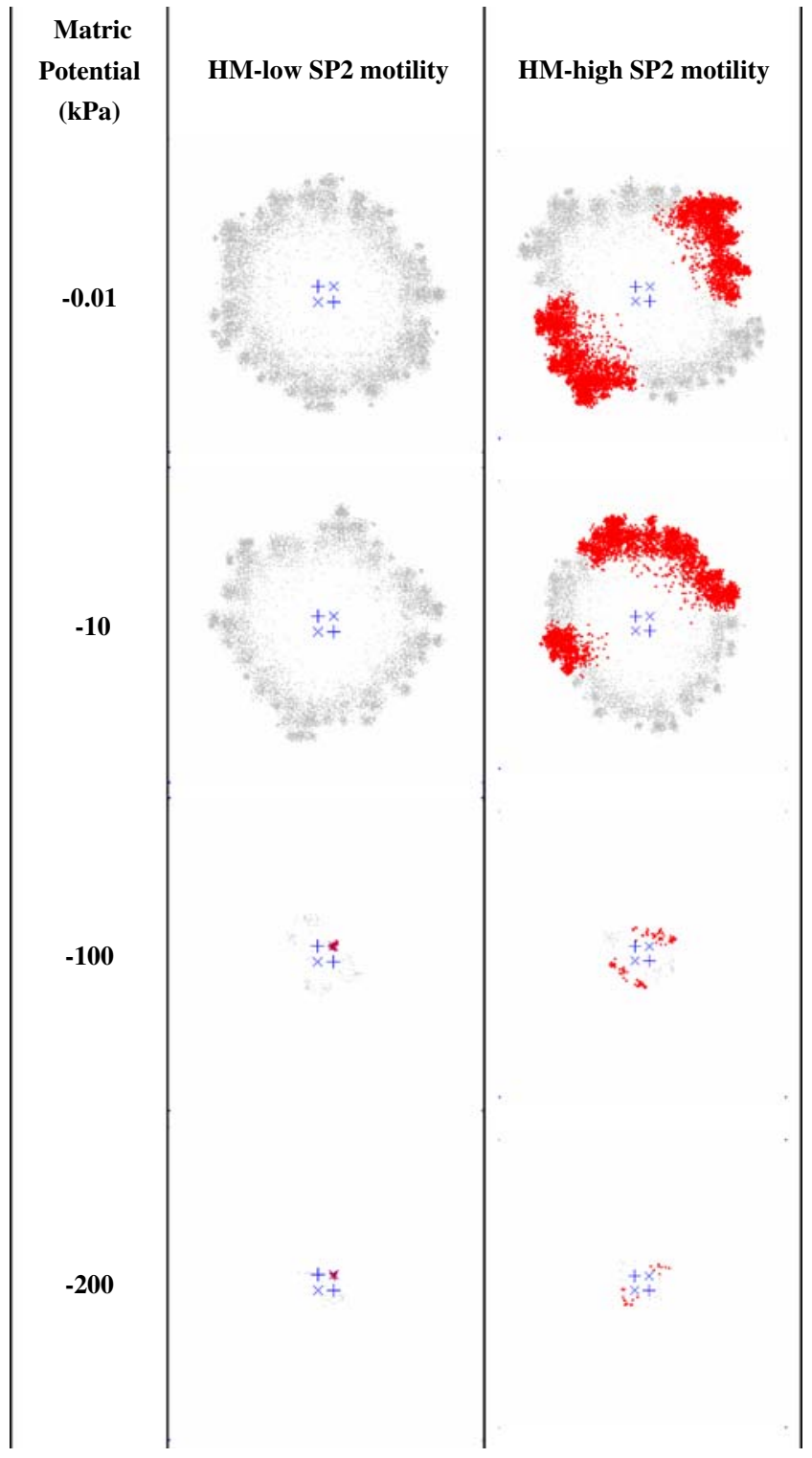

Figure 8 The shape of microbial colonies after $150 \mathrm{~h}$ of simulation time under four different matric potential values. The gray dots represent cells of SP1, and the red ones represent SP2. The left column used the original motility parameters, and the right column adopted the increased SP2 motility. "+" and " $\times$ " represent the initial inoculation sites

(Fig. 11a, b, respectively). Considering the wide range of possible growth curves populating the space between the highest and lowest matric potential values (estimated by the shaded area in Fig. 11, based on five replicates under constant matric potential of -10 and $-100 \mathrm{kPa}$ ), growth curves predicted using $\bar{D}_{\mathrm{e}}$ values provide reasonable approximation of behavior under alternating hydration conditions, except for exceedingly long dry periods where even SP1 became extinct.

In contrast, simulations for the less competitive species SP2 yielded growth curves that did not reflect synchronized patterns with the variations in matric potential. Moreover, simulated growth of SP2 under mean effective diffusion values did not match growth under the fluctuating hydration conditions. This lack of agreement could be attributed to the critical role of competitive nutrient interception by SP1 that controls the nutrient availability for SP2. These findings lend support to the existence of a key physical (diffusion-based) parameter that captures the diffusional capacity of a rough surface with respect to microbial growth and activity. A similar finding was deduced in previous studies for microbial growth on rough surface network [20].

\section{Discussion}

The simulation results suggest that drier conditions promote the coexistence of two competing microbial species due to limited motility [28] and spatial variations in resource
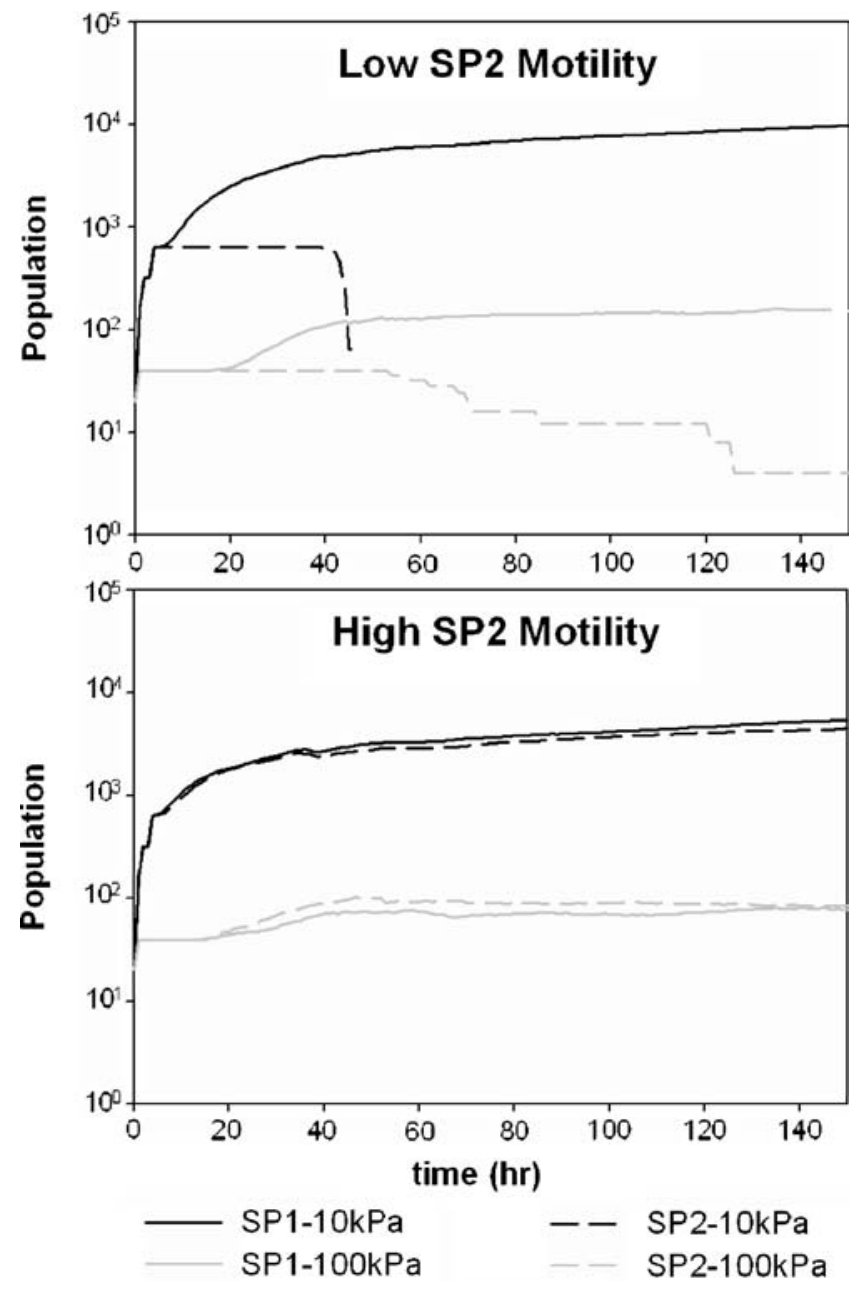

Figure 9 Population curves for the 150-h simulation time for the -10 and -100 kPA scenarios demonstrated in Fig. 8 


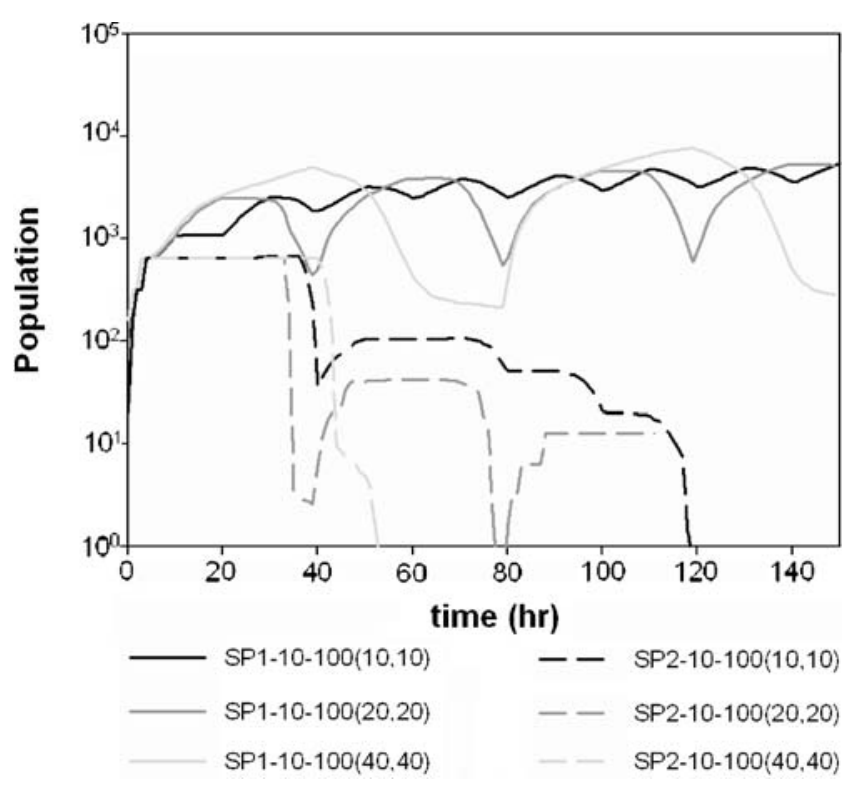

Figure 10 Population growth curves for temporal variations of matric potentials with alternating and equal wet and dry periods 10,20 , and $40 \mathrm{~h}$ each

availability. Under temporal variations in matric potential values similar to those induced by intermittent rainfall or irrigation, temporal average effective diffusion coefficient of the rough surface (and by extrapolation, in unsaturated soils) may be used to estimate activity and growth of dominant microbial species as controlled by the diffusional capacity of the system.

Although simulations were based on a specific set of parameters and required many simplifying assumptions, results capture similar trends predicted in other studies and may be used to guide future experiments and to identify and quantify trends concerning microbial behavior in unsaturated soils. The spatial heterogeneity of resource distribution in soils defines a range of habitats and shapes the growth and abundance of microbial species. Restricted mobility under drier conditions deprive motile species of the advantage of fast growth or rapid spreading, hence forces all the species to receive resources passively based on diffusion into new habitats. Under these limiting conditions, the spatial position and location of a habitat (in the context of nutrient availability) becomes more important for species survival rather than their physiological advantages. This phenomena captured in our simulations may help explain observations [34] of higher microbial diversity in drier soil, where prospects for spreading of either microbes or resources are limited, and geological distribution of resources predetermines the suitable locations for growth. Under wetter condition, aqueous phase is more connected and distribution of microbes and resources will be more homogenized, which increases the intensity of competition.

Temporal variations and spatial heterogeneity of soil hydration status are critical for promoting and preserving coexistence. Although low water content reduces overall available nutrient originally held in the aqueous phase and
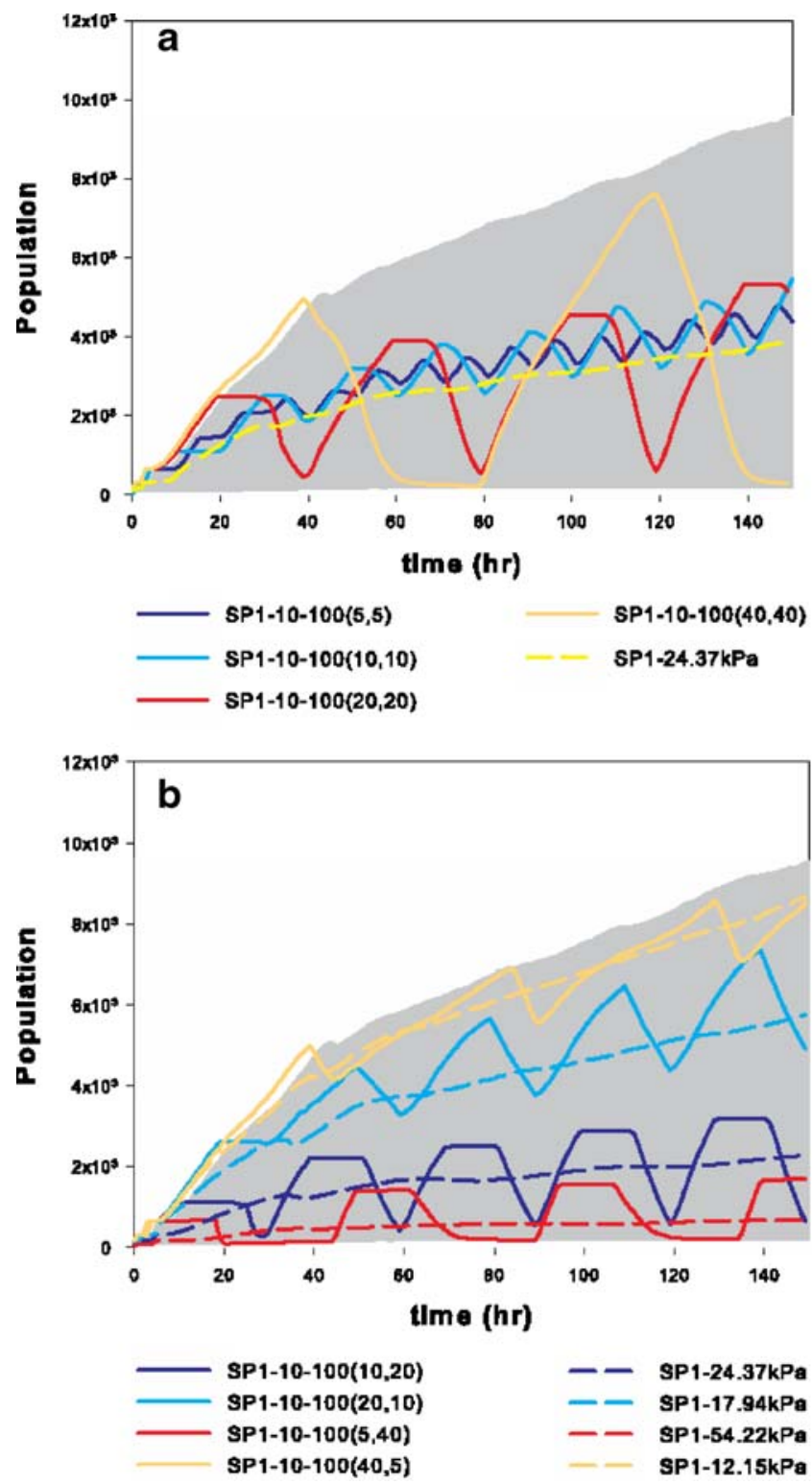

Figure 11 Population growth curves of SP1 under matric potential alternations between -10 and $-100 \mathrm{kPa}$ with equal durations of the wet and dry periods (top) and nonequal durations (bottom). The shaded area encloses the range of growth curves between -10 and $-100 \mathrm{kPa}$ (obtained from five replicates of growth curves under the limiting matric potentials, respectively). a Dashed line is the growth curve under steady $-24 \mathrm{kPa}$, which is the matric potential corresponding to the temporal mean effective diffusion coefficient $\bar{D}_{\mathrm{e}}$ (Eq. 7). b Dashed lines corresponding to the $\bar{D}_{\mathrm{e}}$ values corresponding to the varied potential scenarios, which are plotted in solid lines with the same color 
nutrient fluxes supplied through the aqueous diffusional pathways, coexistence of the two species was considerably prolonged under a range of drier and diffusion-limited environment primarily due to constraints on mobility which delayed spatial intermingling of species. Although certain biological parameters were chosen to enhance and highlight competitive differences of the two species, the prolonged coexistence time due to limited transport and diffusion capacity significantly contributed to the maintenance of microbial diversity. This coexistence was supported also by variable dynamic processes, which although do not lead to equilibrium states [16], allowed weaker species to survive until conditions became more favorable. Additionally, under many natural conditions, a dominant species may not attain very high growth rates and motility as assumed in our simulations, and species coexistence would be easier to maintain under less intensive competition.

The simulation results also captured the impact of transport properties of the porous medium on microbial growth and coexistence at a larger scale (in this case, effective $D_{\mathrm{e}}$ for the entire rough surface) compared with the size of microbial habitats. Larger-scale transport capacity imposes an overall control of total population by limiting the available nutrient flux. It also controls the magnitude of competition if microbial motility is related with aqueous abundance. As shown previously, temporal variations in the domain-scale effective diffusion coefficient corresponded closely to the variations in population of the dominant species. The temporally averaged effective diffusion coefficient $\bar{D}_{\mathrm{e}}$ could satisfactorily indicate the growth tendency of the dominant species under complicated temporal variation in matric potential. These findings highlighted the need for considering critical bulk transport properties when studying soil microbial dynamics. For example, for high soil water contents, gaseous diffusion and oxygen may become the limiting factor for microbial growth [29].

In conclusion, the proposed modeling approach provided a mathematically and physically tractable framework for considering environmental and biological parameters and yielded new insights into microbial ecosystem evolution under a range of conditions found in soils. The model is also expandable to address more complex chemical and biological species and reactions. The simplified simulated scenarios involving basic competition of two species for a single resource are not very common in nature, while biological parameters are scarce and difficult to quantify. Nevertheless, experimental studies are underway to address some of the quantitative challenges under well-controlled artificial rough surfaces using pure cultures to test some of the predicted trends and provide more robust parameters for more refined quantitative modeling of microbial coexistence in unsaturated soils $[11,12]$.

\section{Appendix}

List of symbols

$C$ solute concentration

$C_{0}$ a constant concentration

$D$ local aqueous diffusion coefficient

$D_{0}$ solute diffusion coefficient in aqueous phase

$D_{\text {e }}$ effective diffusion coefficient

$\bar{D}_{\text {e temporally averaged diffusion coefficient }}$

$D_{\text {e,dry }}$ effective diffusion coefficient in the dry period

$D_{\text {e,wet }}$ effective diffusion coefficient in the wet period

$D_{\mathrm{S}}$ effective diffusion coefficient in the unsaturated rough surface

$K$ s half-saturation constant

$m$ an empirical parameter in the van Genuchten model

$N$ concentration of nutrient

$n$ an empirical parameter in the van Genuchten model

$R$ local consumption rate of nutrient

$R^{*}$ nutrient concentration at $\mu=0$

$r$ cell motility

$r$ radial distance

$r_{\mathrm{m}}$ apparent maintenance rate at $\mu=0$

$s$ substrate concentration

$t$ variable of time

$t_{\text {dry }}$ duration of the dry period

$t_{\text {wet }}$ duration of the wet period

$V$ aqueous volume of the lattice grid

$V_{\max }$ maximum specific substrate uptake rate

$\overline{V_{\mu}}$ median cell volume at $\mu=0$

$v$ rate of substrate uptake

$x$ cell dry mass of the individual cell

$Y_{\max }$ apparent yield at $\mu_{\max }$, corrected for maintenance

$\alpha$ a parameter in the van Genuchten model

$\Delta M_{1}$ amount of added nutrient at the boundaries to maintain constant concentration

$\Delta M_{2}$ amount of removed nutrient at the center to maintain constant concentration

$\Theta$ effective water content in the van Genuchten model

$\theta$ volumetric water content

$\theta_{\mathrm{r}}$ residual water content

$\theta_{\mathrm{s}}$ volumetric water content at saturation

$\mu$ specific growth rate

$\mu_{\max }$ maximum specific growth rate

$\rho$ cell density (based on dry mass)

$\Phi$ rough surface porosity

$\psi_{\mathrm{m}}$ matric potential

Acknowledgements The authors gratefully acknowledge the partial support of BARD-US-Israel Binational Agricultural Research and Development Fund under grant US-3377-02, the National Science Foundation (Hydrologic Sciences) under grant EAR-0409364, and 
funding by the Swiss National Science Foundation project 200021113442. We thank Hannes Fluhler (ETH, Zurich); Shmulik Friedman (ARO-Volcani, Israel); and Barth Smets and Arnaud Dechesne (DTU, Copenhagen) for the stimulating discussions and assistance with various aspects of this work. We sincerely appreciate numerous valuable comments and suggestions offered by the editor and three anonymous reviewers.

\section{References}

1. Batterman S, Padmanabham I, Milne P (1996) Effective gas-phase diffusion coefficients in soils at varying water content measured using a one-flow sorbent based technique. Environ Sci Technol 30:770-778

2. Berg HC (2005) Swarming motility: it better be wet. Curr Biol 15: R599-R600

3. Biondi SA, Quinn JA, Goldfine H (1998) Random motility of swimming bacteria in restricted geometries. AIChE J 44:19231929

4. Bird RB, Stewart WE, Lightfoot EN (2002) Transport phenomena. Wiley, New York

5. Brooks RH, Corey AT (1964) Hydraulic properties of porous media. Colorado State University, Fort Collins, Colorado

6. Broseta D, Barre L, Vizika O, Shahidzadeh N, Guilbaud JP, Lyonnard S (2001) Capillary condensation in a fractal porous medium. Phys Rev Lett 86:5313-5316

7. Brown SR (1987) A note on the description of surface-roughness using fractal dimension. Geophys Res Lett 14:1095-1098

8. Chen KC, Ford RM, Cummings PT (1998) Mathematical models for motile bacterial transport in cylindrical tubes. J Theor Biol 195:481-504

9. Curtis TP, Sloan WT, Scannell JW (2002) From the cover: estimating prokaryotic diversity and its limits. Proc Natl Acad Sci U S A 99:10494-10499

10. Darnton NC, Turner L, Rojevsky S, Berg HC (2007) On torque and tumbling in swimming Escherichia coli. J Bacteriol 189:1756-1764

11. Dechesne A, Or D, Gulez G, Smets BF (2008) The porous surface model, a novel experimental system for online quantitative observation of microbial processes under unsaturated conditions. Appl Environ Microbiol 74:5195-5200

12. Dechesne A, Or D, Smets BF (2008) Limited diffusive fluxes of substrate facilitate coexistence of two competing bacterial strains. FEMS Microbiol Ecol 64:1-8

13. Dens EJ, Van Impe JF (2000) On the importance of taking space into account when modeling microbial competition in structured food products. Math Comput Simul 53:443-448

14. Fredrickson AG, Stephanopoulos G (1981) Microbial competition. Science 213:972-979

15. Harshey RM (2003) Bacterial motility on a surface: many ways to a common goal. Annu Rev Microbiol 57:249-273

16. Hutchinson GE (1961) The paradox of the plankton. Am Nat 95:137-145

17. Kreft JU, Booth G, Wimpenny JWT (1998) Bacsim, a simulator for individual-based modelling of bacterial colony growth. Microbiology 144:3275-3287

18. Liu HH (2004) A constitutive-relationship model for film flow on rough fracture surfaces. Hydrogeol J 12:237-240
19. Long T, Or D (2005) Aquatic habitats and diffusion constraints affecting microbial coexistence in unsaturated porous media. Water Resour Res 41:W08408. doi:08410.01029/02004W R003796

20. Long T, Or D (2007) Microbial growth on partially saturated rough surfaces: simulations in idealized roughness networks. Water Resour Res 43:W02409. doi:02410.01029/02005 WR004781

21. Moldrup P, Olesen T, Schjonning P, Yamaguchi T, Rolston DE (2000) Predicting the gas diffusion coefficient in undisturbed soil from soil water characteristics. Soil Sci Soc Am J 64:94-100

22. Olson MS, Ford RM, Smith JA, Fernandez EJ (2005) Analysis of column tortuosity for $\mathrm{mncl} 2$ and bacterial diffusion using magnetic resonance imaging. Environ Sci Technol 39:149-154

23. Or D, Hanks RJ (1992) Soil-water and crop yield spatial variability induced by irrigation nonuniformity. Soil Sci Soc Am J 56:226-233

24. Or D, Smets BF, Wraith JM, Dechesne A, Friedman SP (2007) Physical constraints affecting bacterial habitats and activity in unsaturated porous media-a review. Adv Water Resour 30:15051527

25. Or D, Tuller M (2000) Flow in unsaturated fractured porous media: hydraulic conductivity of rough surfaces. Water Resour Res 36:1165-1177

26. Press WH, Flannery BP, Teukolsky SA, Vetterling WT (1986) Numerical recipes: the art of scientific computing. Cambridge University Press, Cambridge

27. Radlinski AP, Radlinska EZ, Agamalian M, Wignall GD, Lindner P, Randl OG (1999) Fractal geometry of rocks. Phys Rev Lett 82:3078-3081

28. Reichenbach T, Mobilia M, Frey E (2007) Mobility promotes and jeopardizes biodiversity in rock-paper-scissors games. Nature 448:1046-1049

29. Skopp J, Jawson MD, Doran JW (1990) Steady-state aerobic microbial activity as a function of soil water content. Soil Sci Soc Am J 54:1619-1625

30. Tilman D (1982) Resource competition and community structure. Princeton University Press, Princeton, NJ

31. Tokunaga TK, Wan JM, Sutton SR (2000) Transient film flow on rough fracture surfaces. Water Resour Res 36:1737-1746

32. Torsvik V, Ovreas L (2002) Microbial diversity and function in soil: from genes to ecosystems. Curr Opin Microbiol 5:240-245

33. Torsvik V, Ovreas L, Thingstad TF (2002) Prokaryotic diversitymagnitude, dynamics, and controlling factors. Science 296:10641066

34. Treves DS, Xia B, Zhou J, Tiedje JM (2003) A two-species test of the hypothesis that spatial isolation influences microbial diversity in soil. Microb Ecol 45:20-28

35. Tuller M, Or D (2005) Water films and scaling of soil characteristic curves at low water contents. Water Resour Res 41:W09403. doi:09410.01029/02005WR004142

36. van Genuchten MT (1980) A closed-form equation for predicting the hydraulic conductivity of unsaturated soils. Soil Sci Soc Am J 44:892-898

37. Zhou J, Xia B, Treves DS, Wu L-Y, Marsh TL, O'Neill RV, Palumbo AV, Tiedje JM (2002) Spatial and resource factors influencing high microbial diversity in soil. Appl Environ Microbiol 68:326-334

38. Zhou JZ, Xia BC, Huang H, Palumbo AV, Tiedje JM (2004) Microbial diversity and heterogeneity in sandy subsurface soils. Appl Environ Microbiol 70:1723-1734 